\title{
Deterministic rendezvous with detection using beeps *
}

\author{
Samir Elouasbi $\quad$ Andrzej Pelc ${ }^{\ddagger}$
}

\begin{abstract}
Two mobile agents, starting at arbitrary, possibly different times from arbitrary nodes of an unknown network, have to meet at some node. Agents move in synchronous rounds: in each round an agent can either stay at the current node or move to one of its neighbors. Agents have different labels which are positive integers. Each agent knows its own label, but not the label of the other agent. In traditional formulations of the rendezvous problem, meeting is accomplished when the agents get to the same node in the same round. We want to achieve a more demanding goal, called rendezvous with detection: agents must become aware that the meeting is accomplished, simultaneously declare this and stop. This awareness depends on how an agent can communicate to the other agent its presence at a node. We use two variations of the arguably weakest model of communication, called the beeping model, introduced in [8]. In each round an agent can either listen or beep. In the local beeping model, an agent hears a beep in a round if it listens in this round and if the other agent is at the same node and beeps. In the global beeping model, an agent hears a loud beep in a round if it listens in this round and if the other agent is at the same node and beeps, and it hears a soft beep in a round if it listens in this round and if the other agent is at some other node and beeps.

We first present a deterministic algorithm of rendezvous with detection working, even for the local beeping model, in an arbitrary unknown network in time polynomial in the size of the network and in the length of the smaller label (i.e., in the logarithm of this label). However, in this algorithm, agents spend a lot of energy: the number of moves that an agent must make, is proportional to the time of rendezvous. It is thus natural to ask if bounded-energy agents, i.e., agents that can make at most $c$ moves, for some integer $c$, can always achieve rendezvous with detection as well. This is impossible for some networks of unbounded size. Hence we rephrase the question: Can bounded-energy agents always achieve rendezvous with detection in boundedsize networks? We prove that the answer to this question is positive, even in the local beeping model but, perhaps surprisingly, this ability comes at a steep price of time: the meeting time of bounded-energy agents is exponentially larger than that of unrestricted agents. By contrast, we show an algorithm for rendezvous with detection in the global beeping model that works for bounded-energy agents (in bounded-size networks) as fast as for unrestricted agents.
\end{abstract}

Keywords: algorithms, rendezvous, detection, synchronous, deterministic, network, graph,beep.

\footnotetext{
${ }^{*}$ A preliminary version of this paper appeared in Proc. 11th International Symposium on Algorithms and Experiments for Wireless Sensor Networks (ALGOSENSORS 2015), LNCS 9536, 85-97.

${ }^{\dagger}$ Département d'informatique, Université du Québec en Outaouais, Gatineau, Québec J8X 3X7, Canada. E-mail: elos02@uqo.ca.

${ }^{\ddagger}$ Département d'informatique, Université du Québec en Outaouais, Gatineau, Québec J8X 3X7, Canada. E-mail: pelc@uqo.ca. Supported in part by NSERC discovery grant 8136 - 2013 and by the Research Chair in Distributed Computing of the Université du Québec en Outaouais.
} 


\section{Introduction}

The background and the problem. Two mobile agents, starting at arbitrary, possibly different times from arbitrary nodes of an unknown network, have to meet at some node of it. This task is known as rendezvous [2]. The network is modeled as a simple undirected connected graph, and agents move in synchronous rounds: in each round an agent can either stay at the current node or move to one of its neighbors. Hence in each round an agent is at a specific node. Agents are mobile entities with unlimited memory; from the computational point of view they are modeled as Turing machines. In applications, these entities may represent mobile robots navigating in a labyrinth or in corridors of a building, or software agents moving in a communication network. The purpose of meeting might be to exchange data previously collected by the agents at nodes of the network, or to coordinate future network maintenance tasks, for example checking functionality of websites or of sensors connected in a network.

Agents have different labels which are positive integers. Each agent knows its own label, but not the label of the other agent. Agents do not know the topology of the network, they do not have any bound on its size. They do not know the starting node or activation time of the other agent. They cannot mark the visited nodes in any way. Each agent appears at its starting node at the time of its activation by the adversary.

We seek rendezvous algorithms that do not rely on the knowledge of node labels, and can work in anonymous networks as well (cf. [2]). The importance of designing such algorithms is motivated by the fact that, even when nodes are equipped with distinct labels, agents may be unable to perceive them because of limited sensory capabilities, or nodes may refuse to reveal their labels to agents, e.g., due to security or privacy reasons. On the other hand, we assume that edges incident to a node $v$ have distinct labels in $\{0, \ldots, d-1\}$, where $d$ is the degree of $v$. Thus every undirected edge $\{u, v\}$ has two labels, which are called its port numbers at $u$ and at $v$. Port numbering is local, i.e., there is no relation between port numbers at $u$ and at $v$. An agent entering a node learns the port of entry and the degree of the node. Note that, in the absence of port numbers, rendezvous is usually impossible, as all ports at a node look identical to an agent and the adversary may prevent the agent from taking some edge incident to the current node.

In traditional formulations of the rendezvous problem, meeting is accomplished when the agents get to the same node in the same round. We want to achieve a more demanding goal, called rendezvous with detection: agents must become aware that the meeting is accomplished, simultaneously declare this and stop. This awareness depends on how an agent can communicate to the other agent its presence at a node. We use two variations of the beeping model of communication. In each round an agent can either listen, i.e., stay silent, or beep, i.e., emit a signal. In the local beeping model, an agent hears a beep in a round if it listens in this round and if the other agent is at the same node and beeps. In the global beeping model, an agent hears a loud beep in a round if it listens in this round and if the other agent is at the same node and beeps, and it hears a soft beep in a round if it listens in this round and if the other agent is at some other node and beeps.

The beeping model has been introduced in [8] for vertex coloring, and used in [1] to solve the MIS problem, and in [23] to construct a minimum connected dominating set. In [15], the authors studied the quantity of computational resources needed to solve problems in complete networks 
using beeps. In the variant from the above papers the beeping entities were nodes rather than agents, and beeps of a node were heard at adjacent nodes. The beeping model is widely applicable, as it makes small demands on communicating devices, relying only on carrier sensing. In the case of mobile agents, both the local and the global beeping models are applicable in different settings. The local model is applicable even for agents having very weak transmissions capabilities, limiting reception of a beep to the same node. The global model is applicable for more powerful agents, that can beep sufficiently strongly to be heard in the entire network, and having a listening capability of differentiating a beep emitted at the same node from a beep emitted at a different node.

It should be noted that our local beeping model is arguably the weakest way of communication between agents: they can communicate only when residing simultaneously at the same node, they cannot hear when they beep, and messages are the simplest possible. In fact, as mentioned in [8], local beeps are an even weaker way of communicating than using one-bit messages, as the latter ones allow three different states ( 0,1 and no message), while local beeps permit to differentiate only between a signal and its absence. Clearly, without any communication, rendezvous with detection is impossible, as agents cannot become aware of each other's presence at a node. Notice also that in the global beeping model it would not be possible to remove the distinction between hearing a loud beep when the beeping agent is at the same node and hearing a soft beep when the beeping agent is at a different node. Indeed, the same strength of beep reception would make it impossible for an agent $A_{1}$ to inform the other agent $A_{2}$ of the presence of $A_{1}$ at the same node, and hence rendezvous with detection would be impossible. The global beeping model is at least as strong as the local one, in the sense that any algorithm of rendezvous with detection working in the local model works also in the global model, by simply ignoring soft beeps. We will see that the converse is not true.

For a given network, the execution time of an algorithm of rendezvous with detection, for agents with given labels starting in given rounds from given initial positions, is the number of rounds from the activation of the later agent to the declaration of rendezvous. For a given class of networks, the time of an algorithm of rendezvous with detection is its worst-case execution time, over all networks in the class, all initial positions, all pairs of distinct labels and all starting times.

Our results. Our first result answers the basic question: Is it possible to achieve rendezvous with detection in arbitrary networks, and if so, how fast it can be done? We present a deterministic algorithm of rendezvous with detection working, even for the local beeping model, in an arbitrary unknown network in time polynomial in the size of the network and in the length of the smaller label (i.e., in the logarithm of this label). In fact, the time complexity of our algorithm matches that of the fastest, known to date, rendezvous algorithm without detection, constructed in [22].

However, in this algorithm, agents spend a lot of energy: the number of moves that an agent must make, is proportional to the time of rendezvous. On the other hand, in many applications, e.g., when agents are mobile robots, they are battery-powered devices, and hence the energy that an agent can spend on moves is limited. It is thus natural to ask if bounded-energy agents, i.e., agents that can make at most $c$ moves, for some integer $c$, can always achieve rendezvous with detection as well. This is impossible for some networks of unbounded size. Hence we rephrase the question: Can bounded-energy agents always achieve rendezvous with detection in bounded-size networks? We prove that the answer to this question is positive, even in the local beeping model but, perhaps 
surprisingly, this ability comes at a steep price of time: the meeting time of bounded-energy agents is exponentially larger than that of unrestricted agents. By contrast, we show an algorithm for rendezvous with detection in the global beeping model that works for bounded-energy agents (in bounded-size networks) as fast as for unrestricted agents.

Related work. The vast literature on rendezvous can be divided according to the mode in which agents move (deterministic or randomized) and the environment where they move (a network modeled as a graph or a terrain in the plane). An extensive survey of randomized rendezvous in various scenarios can be found in [2], cf. also [3, 17]. Rendezvous of two or more agents in the plane has been considered e.g., in [12, 13].

Our paper is concerned with deterministic rendezvous in networks, surveyed in [20]. In this setting a lot of effort has been dedicated to the study of the feasibility of rendezvous, and to the time required to achieve this task, when feasible. For instance, deterministic rendezvous with agents equipped with tokens used to mark nodes was considered, e.g., in [18]. Time of deterministic rendezvous of agents equipped with unique labels was discussed in [10, 22]. Memory required by the agents to achieve deterministic rendezvous has been studied in [4, 14] for trees and in [9] for general graphs. In [19] the authors studied tradeoffs between the time of rendezvous and the total number of edge traversals by both agents until the meeting.

Apart from the synchronous model used in this paper, several authors have investigated asynchronous rendezvous in the plane [7, 12, 13] and in network environments [5, 11]. In the latter scenario the agent chooses the edge which it decides to traverse but the adversary controls the speed of the agent. Under this assumption rendezvous in a node cannot be guaranteed even in very simple graphs, and hence the rendezvous requirement is relaxed to permit the agents to meet inside an edge.

\section{Preliminaries}

In the rest of the paper the word "graph" means a simple connected undirected graph modeling a network. The size of a graph is the number of its nodes. In this section we recall two procedures known from the literature, that will be used as building blocks in our algorithms. The aim of the first procedure is graph exploration, i.e., visiting all nodes of a graph by a single agent. The procedure, called $\operatorname{EXP}(m)$, is based on universal exploration sequences (UXS) [16], and follows from the result of Reingold [21. Given any positive integer $m$, it allows the agent to visit all nodes of any graph of size at most $m$, starting from any node of this graph, using $R(m)$ edge traversals, where $R$ is some polynomial.

A UXS is an infinite sequence $x_{1}, x_{2}, \ldots$ of non-negative integers. Given this sequence, whose effective construction follows from [16, 21], the procedure $\operatorname{EXP}(m)$ can be described as follows. In step 1 , the agent leaves the starting node by port 0 . For $i \geq 1$, the agent that entered the current node of degree $d$ by some port $p$ in step $i$, computes the port $q$ by which it has to exit in step $i+1$ as follows: $q=\left(p+x_{i}\right) \bmod d$. The result of Reingold implies that if an agent starts at any node

$v$ of an arbitrary graph with at most $m$ nodes, and applies procedure $\operatorname{EXP}(m)$, then it will visit all nodes of the graph after $R(m)$ steps. 
The second procedure, due to Ta-Shma and Zwick [22], guarantees rendezvous (without detection) in an arbitrary graph. Below we briefly sketch this procedure, which will be used in our algorithm of rendezvous with detection for unrestricted agents.

Let $\mathbb{Z}^{+}$denote the set of positive integers and let $\mathbb{Z}^{*}$ denote the set of integers greater or equal than -1 . For any positive integer $L$, Ta-Shma and Zwick define a function $\Phi_{L}: \mathbb{Z}^{+} \times \mathbb{Z}^{+} \times \mathbb{Z}^{*} \longrightarrow \mathbb{Z}^{*}$. Intuitively, this function describes a walk in the graph: when an agent starts at some node $v$ of the graph, the function $\Phi$ indicates which port the agent should take in the $t$-th step of its walk, or that it should stay idle (i.e., do not move) in the $t$ th step. The three parameters of the function are: the next step number $t$, the degree $d$ of the current node, and the port number $p$ by which the agent entered the current node in the previous step, or -1 if it stayed idle in the previous step. The value of the function is either the port number by which the agent should leave the current node in the next step, or -1 if it should stay in the next step.

More formally, the function $\Phi_{L}$ is applied by an agent with label $L$ in a graph $G$ at a node $v$ of $G$ as follows. Let $v_{0}=v$ and let $v_{1}$ be the node adjacent to $v_{0}$, such that the edge $\left\{v_{0}, v_{1}\right\}$ has port number 0 at $v_{0}$. Suppose that nodes $v_{0}, v_{1}, \ldots, v_{t-1}$ are already constructed, so that $v_{i+1}$ either equals $v_{i}$ or is adjacent to $v_{i}$. The node $v_{t}$ is defined as follows. In the case when $v_{t-1}=v_{t-2}$ and the degree of $v_{t-1}$ is $d$, then $v_{t}=v_{t-1}$ if $\Phi_{L}(t, d,-1)=-1$; if $\Phi_{L}(t, d,-1)=q \geq 0$ then $v_{t}$ is the node adjacent to $v_{t-1}$ such that the port number at $v_{t-1}$ corresponding to edge $\left\{v_{t-1}, v_{t}\right\}$ is $q$. In the case when $v_{t-1} \neq v_{t-2}$, the port number at $v_{t-1}$ corresponding to edge $\left\{v_{t-1}, v_{t-2}\right\}$ is $p$ and the degree of $v_{t-1}$ is $d$, then $v_{t}=v_{t-1}$ if $\Phi_{L}(t, d, p)=-1$; if $\Phi_{L}(t, d, p)=q>0$ then $v_{t}$ is the node adjacent to $v_{t-1}$ such that the port number at $v_{t-1}$ corresponding to edge $\left\{v_{t-1}, v_{t}\right\}$ is $q$. Hence the application of function $\Phi_{L}$ at node $v$ defines an infinite walk of the agent with label $L$ in the graph $G$. This walk starts at $v$ and in each round $t$ the agent either stays at the current node or moves to an adjacent node by a port determined by the function $\Phi_{L}$ on the basis of the degree of the current node and of the port by which the agent entered it. A round $t$ is called active for the agent if $v_{t} \neq v_{t-1}$ and it is called passive if $v_{t}=v_{t-1}$.

The following result, proved in [22], guarantees rendezvous without detection in polynomial time, if two agents apply functions $\Phi_{L}$ corresponding to their labels, in an unknown graph.

Theorem 2.1 There exists a polynomial $P$ in two variables, with the following property. Let $G$ be an n-node graph and consider two agents with distinct labels $L_{1}, L_{2}$ respectively, starting at nodes $v$ and $w$ of the graph in rounds $t_{1} \geq t_{2}$. Let $t \geq t_{1}$ and let $\ell$ be the smaller label. If agent with label $L_{i}$ applies function $\Phi_{L_{i}}$ at its starting node, for $i=1,2$, then agents are simultaneously at the same node in some round of the time interval $[t, t+P(n, \log \ell)]$. Moreover, rendezvous occurs in a round which is active for one of the agents and passive for the other. The same property remains true if one of the agents stays idle and the other agent applies its function $\Phi_{L_{i}}$.

\section{Rendezvous with detection of unrestricted agents}

In this section we describe and analyze an algorithm of rendezvous with detection which works for unrestricted agents, i.e., for agents that can spend an arbitrary amount of energy on moves. It works even for the weaker of our two models, i.e., for the local beeping model. Our algorithm uses 
the following procedure which describes an infinite walk of an agent with label $L$, based on the above described application of the function $\Phi_{L}$.

\section{Procedure Beeping walk}

Consider an agent with label $L$ starting at node $v$ of a graph $G$. Let $W$ be the walk resulting from the application of $\Phi_{L}$ in graph $G$ at node $v$. Each round of $W$ is replaced by 2 consecutive rounds as follows. If round $t$ of $W$ is passive, i.e., $v_{t}=v_{t-1}$, then this round is replaced by two rounds in which the agent stays at $v_{t}$ and listens. If round $t$ of $W$ is active, i.e., $v_{t} \neq v_{t-1}$, then this round is replaced by the following two rounds: in the first of these rounds the agent goes to $v_{t}$ and beeps, and in the second of these rounds the agent stays at $v_{t}$ and listens.

We now describe our algorithm for rendezvous with detection. It is executed by each agent. Note that the execution of procedure Beeping walk, called by the algorithm, depends on the label of the agent.

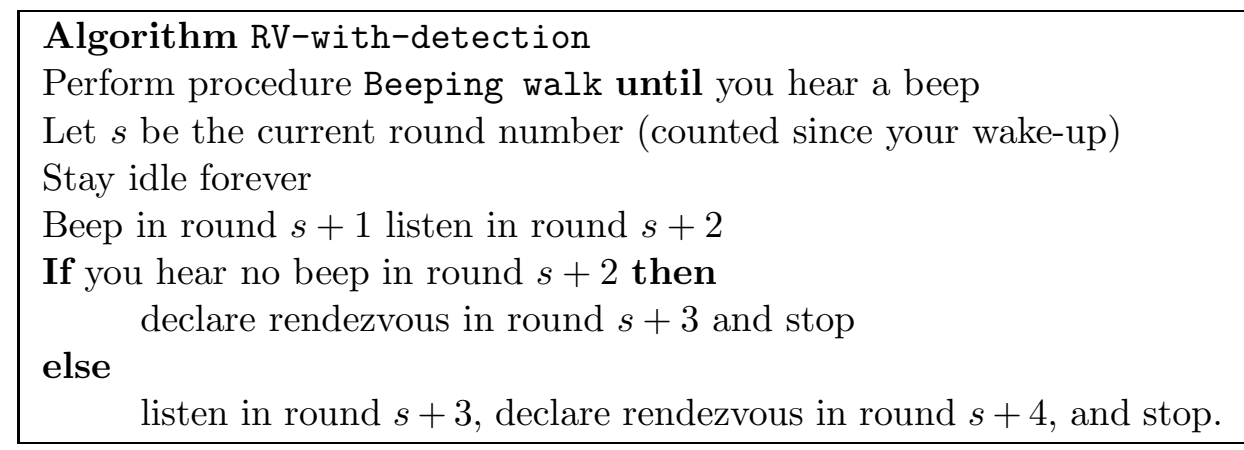

We now show that Algorithm RV-with-detection correctly accomplishes rendezvous with detection and works in time polynomial in the size of the graph and in the logarithm of the smaller label. The agent that starts later will be called the later agent and the other one the earlier agent. If agents start simultaneously, these qualifiers are attributed arbitrarily.

Theorem 3.1 Consider two agents with distinct labels $L_{1}, L_{2}$ respectively, starting at nodes $v$ and $w$ of an n-node graph in possibly different rounds. Let $\ell$ be the smaller label. If both agents execute Algorithm RV-with-detection, then they meet and simultaneously declare rendezvous in time $O(P(n, \log \ell))$, i.e., polynomial in $n$ and in $\log \ell$, after the start of the later agent.

Proof. An agent executing Algorithm RV-with-detection starts by performing procedure Beeping walk. We first prove that when both agents execute this procedure there must be a round in which one of them hears the beep of the other. By Theorem 2.1, when agent with label $L_{i}$ applies function $\Phi_{L_{i}}$ at its starting node, for $i=1,2$, there is a round $r$ when they meet, and this round is active for one agent and passive for the other. (The names of the rounds are for the ease of description only, as none of the agents knows them.) This holds regardless of the starting rounds and starting positions of the agents. By definition, procedure Beeping walk is a simulation of the application of $\Phi_{L_{i}}$ with 2-round segments corresponding to rounds. A segment simulating an active (resp. passive) round will be called an active (resp. passive) segment. 
Suppose that the segment simulating the meeting round $r$ is the $\rho$ th segment of the earlier agent. If the segments of the two agents are aligned, i.e., the first round of the later agent is the first round of a segment of the earlier agent, then in the first round of the $\rho$ th segment of the earlier agent one agent beeps and the other listens because this segment is active for one of them and passive for the other. Since both agents are at the same node in this round, one of them hears the beep of the other. Hence we may assume that the segments are not aligned. Suppose that the $\sigma$ th segment of the later agent starts during the $\rho$ th segment of the earlier agent, i.e., the first round of the $\sigma$ th segment of the later agent is the second round of the $\rho$ th segment of the earlier agent. By Theorem 2.1 there are two cases: either the $\rho$ th segment of the earlier agent is passive and the $\sigma$ th segment of the later agent is active, or the $\rho$ th segment of the earlier agent is active and the $\sigma$ th segment of the later agent is passive. In the first case let $r^{\prime}$ be the first round of the $\sigma$ th segment of the later agent. In round $r^{\prime}$ both agents are at the same node and the later agent beeps while the earlier agent listens, hence it hears the beep. Consider the second case. Regardless of whether the $(\sigma-1)$ th segment of the later agent is active or passive, during this segment the later agent is at the same node as the earlier agent during its $\rho$ th segment. Let $r^{\prime \prime}$ be the first round of the $\rho$ th segment of the earlier agent. Since segments of agents are not aligned, in round $r^{\prime \prime}$, when the earlier agent beeps, the later agent listens, and hence hears the beep. This shows that in all cases there must be a round in which one of the agents hears the beep of the other.

Let $t$ be the first round in the execution of Algorithm RV-with-detection by both agents, in which one agent hears the beep of the other. Denote by $A_{1}$ the agent that listens in round $t$ and by $A_{2}$ the other agent. By the algorithm, agent $A_{1}$ stays idle from round $t$ on, and beeps in round $t+1$. In this round agent $A_{2}$ stays idle at the same node and listens, as it still executes procedure Beeping walk, and this is the second round of a segment for this agent. Hence agent $A_{2}$ hears a beep in round $t+1$. This is the first beep that it hears. Consequently, agent $A_{2}$ stays idle from round $t+1$ on, and beeps in round $t+2$. Hence agent $A_{1}$ hears a beep in round $t+2$. By the algorithm, it listens in round $t+3$, declares rendezvous in round $t+4$ and stops. As for agent $A_{2}$, it listens in round $t+3$ and does not hear a beep in this round. Hence it also declares rendezvous in round $t+4$ and stops.

It follows that in round $t+4$ both agents are at the same node, declare rendezvous and stop. This proves the correctness of Algorithm RV-with-detection. It remains to estimate its time. Round $t$ must occur at most $2 P(n, \log \ell)$ rounds after the start of the later agent. Since simultaneous declaration of rendezvous is in round $t+4$, this proves the theorem.

\section{Rendezvous with detection of bounded-energy agents}

In this section we study rendezvous with detection of agents that can perform a bounded number of moves. Let $c$ be a positive integer. A $c$-bounded agent is defined as an agent that can perform at most $c$ moves. (Notice that we do not restrict the number of beeps; indeed, the amount of energy required to make a move is usually so much larger than the amount of energy required to beep that ignoring the latter seems to be a reasonable approximation of reality in many applications.) Can

$c$-bounded agents, for some integer $c$, perform rendezvous with detection in arbitrary graphs? The 
answer to this question is, of course, negative, even if detection is not required. For any integer $c, c$-bounded agents starting at distance larger than $2 c$ cannot meet because at least one of them would have to make more than $c$ steps. Even if we assume that the initial distance between the agents is 1 , meeting of $c$-bounded agents is impossible in some graphs. Indeed, consider two $n$-node stars whose centers are linked by an edge, with agents starting at the centers of the stars. In the worst case, at least one of the agents must make at least $n-1$ steps before meeting (to find the connecting edge), which is impossible for $c$-bounded agents, when $n$ is large.

Thus, we rephrase the question: Can $c$-bounded agents always achieve rendezvous with detection in bounded-size graphs? More precisely, for any integer $n$, does there exist an integer $c$, such that $c$-bounded agents can achieve rendezvous with detection in all graphs of size at most $n$ ? (Notice that, for example, Algorithm RV-with-detection cannot be used here. In this algorithm, the number of steps performed by an agent with label $L$ is proportional to $P(n, \log L)$, and hence, even when the size of the graph is bounded, this number can be arbitrarily large.) The answer to our question turns out to be positive, even in the local beeping model. Below we describe an algorithm that performs this task.

\subsection{Bounded-energy agents in the local beeping model}

Our algorithm uses the following procedure, for an integer parameter $n$.

Procedure Beeping exploration $(n)$

Let $\operatorname{EXP}(n)$ be the procedure described in Section 2 that permits exploration of all graphs of size at most $n$. Replace each round $r$ of $\operatorname{EXP}(n)$ by three consecutive rounds as follows. If in round $r$ of $\operatorname{EXP}(n)$ the agent takes port $p$ to move to node $w$, then in the first of the three replacing rounds the agent takes port $p$ to move to $w$ and beeps, and in the second and third of the replacing rounds it stays at $w$ and listens.

Hence, in each of the three rounds replacing a round $r$ of $\operatorname{EXP}(n)$, the agent is at the same node in Procedure Beeping exploration $(n)$ as it is in Procedure $\operatorname{EXP}(n)$ in round $r$.

We now describe our algorithm for rendezvous with detection of bounded-energy agents, executed by an agent with label $L$ in a graph of size at most $n$. Recall that $R(n)$ is the execution time of $\operatorname{EXP}(n)$. The idea of the algorithm is the following. Its main part, called block, consists of two executions of Procedure Beeping exploration $(n)$ between which a long waiting period is inserted, during which the agent is silent (it listens) and stays idle. The length of this period depends on the label of the agent. We will prove that, regardless of the delay between the starting times of the agents, an entire execution of Procedure Beeping exploration $(n)$ of one of the agents must either fall within the waiting period of the other agent, or must be executed after both executions of this procedure by the other agent. The block of the algorithm executed by a given agent is interrupted in one of the two cases: either when (a) the agent hears a beep during its waiting period or after completing its block, or when (b) it hears beeps in two consecutive rounds during one of the executions of Procedure Beeping exploration $(n)$. In case (a) the agent responds by beeps in two consecutive rounds, declares rendezvous in the next round and stops. In case (b) it declares rendezvous in the next round and stops. 
Below we give the pseudo-code of the algorithm executed by an agent with label $L$ in a graph of size at most $n$. During the executions of Procedure Beeping exploration $(n)$, a boolean variable waiting is set to false, and during the waiting period and after the second execution of Procedure Beeping exploration $(n)$ it is set to true. We use a boolean valued function condition which takes the variable waiting as input, and returns, after each round, the boolean value of the expression

(waiting and you hear a beep) or ( $\neg$ waiting and you hear beeps in two consecutive rounds).

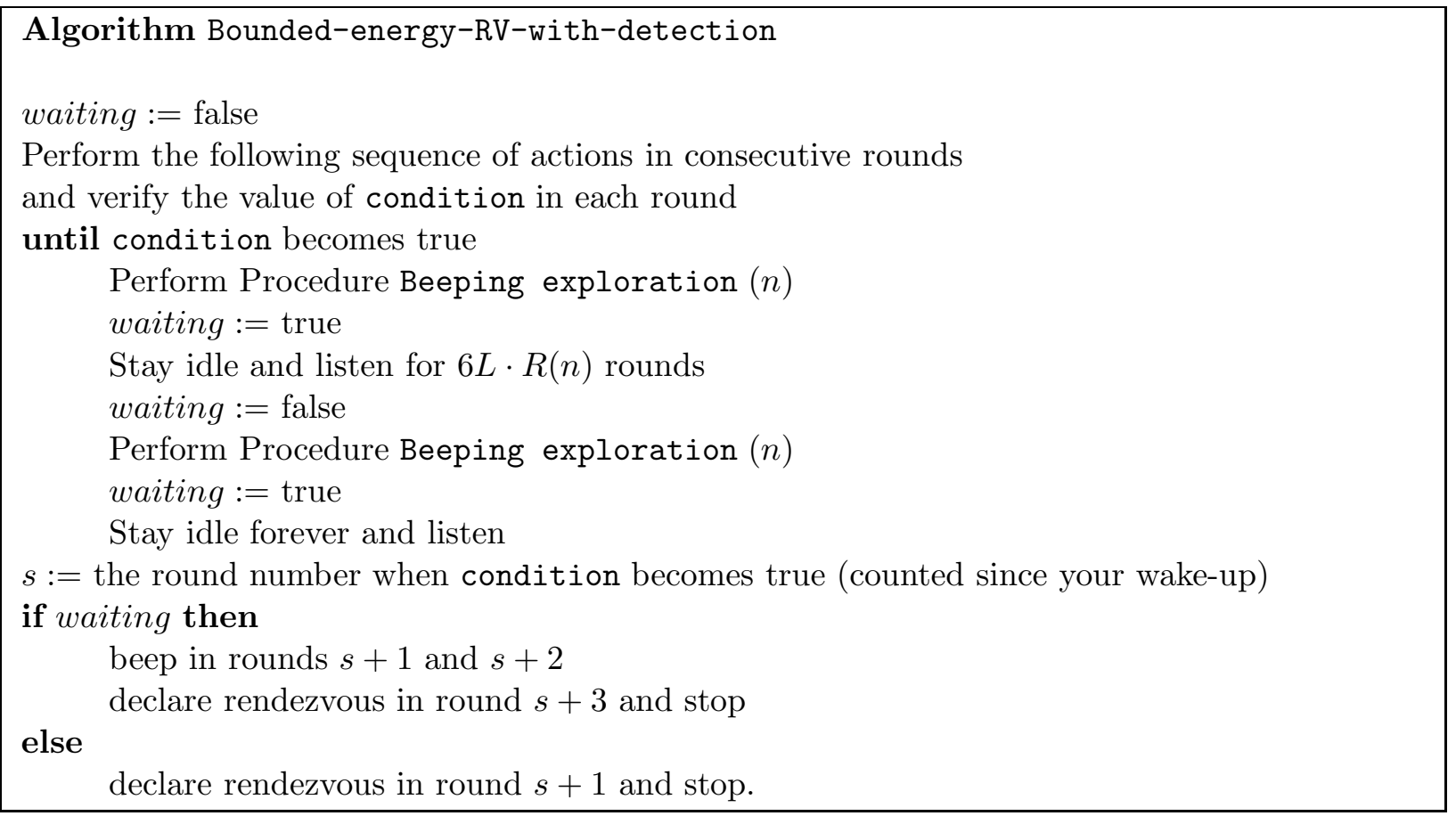

Theorem 4.1 For any positive integer constant $n$ there exists a positive integer $c$, such that Algorithm Bounded-energy-RV-with-detection can be executed by c-bounded agents in any graph of size at most $n$. If two such agents with distinct labels execute this algorithm in such a graph, then they meet and simultaneously declare rendezvous in time $O\left(\ell^{*}\right)$ after the start of the later agent, where $\ell^{*}$ is the larger label.

Proof. Let $n$ be a fixed positive integer. In order to show the existence of the integer $c$, it is enough to show that the number of steps prescribed by the algorithm depends only on the integer $n$ (and not on the label $L$ of the agent). The agent moves only during the two executions of Procedure Beeping exploration $(n)$, at most once every three rounds. Since a full execution of Procedure Beeping exploration $(n)$ takes $E=3 R(n)$ rounds, the agent moves at most $2 E / 3=2 R(n)$ times, and hence it is enough to take $c=2 R(n)$.

We now prove the correctness of the algorithm. Let $B(L)$ be the sequence of actions

Perform Procedure Beeping exploration $(n)$;

Stay idle for $6 L \cdot R(n)$ rounds and listen; (waiting period) 


\section{Perform Procedure Beeping exploration $(n)$;}

For any label $L$, the sequence $B(L)$ of actions will be called the block of the agent with label $L$. We will use the following claim.

Claim. Consider blocks $B\left(L_{1}\right)$ and $B\left(L_{2}\right)$, for $L_{1}>L_{2}$, arbitrarily shifted in time with respect to each other. Then one of the two following properties must hold. Either an entire execution of Procedure Beeping exploration $(n)$ in one of the blocks falls within the waiting period of the other block, or an entire execution of Procedure Beeping exploration $(n)$ in one of the blocks falls after the end of the other block.

In order to prove the claim, consider blocks $B\left(L_{1}\right)$ and $B\left(L_{2}\right)$, for $L_{1}>L_{2}$. Call the block $B\left(L_{1}\right)$ the larger block and the block $B\left(L_{2}\right)$ the smaller block. The waiting period of the smaller block has length $Y=2 E L_{2} \geq 2 E$. Since $L_{1} \geq L_{2}+1$, the waiting period of the larger block has length $2 E L_{1} \geq 2 E\left(L_{2}+1\right)=2 E L_{2}+2 E=Y+2 E$. Let the starting round of the block that starts earlier have number 0 . We will use global round numbers starting from this round. (This is for the purpose of analysis only, as agents do not have access to any global round counter.)

If the smaller block starts earlier, then the second execution of Procedure Beeping exploration $(n)$ of the larger block is performed after the end of the smaller block, and the claim is proved. Hence we may assume that the larger block starts earlier or simultaneously with the smaller block. Let $p$ be the first round of the smaller block. Consider three cases.

Case $1.0 \leq p \leq E$.

In this case the second execution of Procedure Beeping exploration $(n)$ in the smaller block falls entirely within the waiting period of the larger block.

Case 2. $E<p \leq 2 L_{1} E$.

In this case the first execution of Procedure Beeping exploration $(n)$ in the smaller block falls entirely within the waiting period of the larger block.

Case 3. $p>2 L_{1} E$.

In this case the second execution of Procedure Beeping exploration $(n)$ in the smaller block falls entirely after the end of the larger block.

This proves the claim.

Consider agents with labels $L_{1}>L_{2}$. Call the agent with label $L_{1}$ the larger agent and the agent with label $L_{2}$ the smaller agent. The claim implies that there must exist a round $r$ in which condition is satisfied (i.e., has value true) for one of the agents. Indeed, if such a round has not occurred previously, it must occur during the execution of Procedure Beeping exploration $(n)$ by one of the agents, call it $A_{1}$, that falls entirely either in the waiting period of the other agent, call it $A_{2}$, or after the end of the second execution of Procedure Beeping exploration $(n)$ by agent $A_{2}$. In both situations, agent $A_{2}$ has its variable waiting set to true, it is idle and listens during an entire execution of Procedure Beeping exploration $(n)$ by agent $A_{1}$. During this execution, agent $A_{1}$ visits all nodes of the graph and beeps at each node. Hence agent $A_{2}$ hears a beep while its variable waiting is set to true, which means that condition is true for agent $A_{2}$.

Let $r_{0}$ be the first round in which condition is true for some agent. We will show that for this 
agent the first possibility, i.e., (waiting and you hear a beep) must be satisfied in round $r_{0}$. Indeed, suppose that the second possibility, i.e., ( $\neg$ waiting and you hear beeps in two consecutive rounds) is satisfied in round $r_{0}$. These beeps in two consecutive rounds that the agent heard must have been produced in rounds $r_{0}-1$ and $r_{0}$ by the other agent. It could not be during the execution of its block because then the agent beeps only during the execution of Procedure Beeping exploration $(n)$ and this never happens in two consecutive rounds. Hence it must have happened after the block of the other agent has been interrupted. This is possible only when condition has been true for the other agent, which must have occurred before round $r_{0}$, contradicting the definition of this round.

Consider round $r_{0}$ and the agent, call it $A_{3}$, for which the clause (waiting and you hear a beep) is satisfied in round $r_{0}$. Agent $A_{3}$ is at some node $v$ in round $r_{0}$. In round $r_{0}$, the other agent, call it $A_{4}$, must still execute its block, and more precisely it must execute Procedure Beeping exploration $(n)$. It is also at node $v$ in round $r_{0}$. Hence it remains idle at $v$ and listens in the two rounds after it has beeped, i.e., in rounds $r_{0}+1$ and $r_{0}+2$. In these rounds agent $A_{3}$ stays idle at $v$ and beeps. Then it declares rendezvous in round $r_{0}+3$ (still remaining at node $v$ ) and stops. As for agent $A_{4}$, after hearing beeps in rounds $r_{0}+1$ and $r_{0}+2$, the clause ( $\neg$ waiting and you hear beeps in two consecutive rounds) becomes satisfied for it in round $s=r_{0}+2$. Hence agent $A_{4}$ (still remaining at node $v$ ) declares rendezvous in round $s+1=r_{0}+3$ and stops. This concludes the proof of correctness.

It remains to estimate the time between the start of the later starting agent and the declaration of rendezvous. Since $n$ is constant, $R(n)$ is also constant, and hence the duration of the execution of its block by an agent with label $L$ is $(2 L+2) \cdot 3 R(n) \in O(L)$. Let $L_{i}$, where $i=1$, or $i=2$, be the label of the later starting agent. Hence the time between the start of this agent and the declaration of rendezvous is at most $\left(2 L_{i}+2\right) \cdot 3 R(n)+3 \in O\left(L_{i}\right)=O\left(\ell^{*}\right)$ because $L_{i} \leq \ell^{*}$. This concludes the proof.

It is interesting to compare the time sufficient to complete the task of rendezvous with detection, given by Algorithm RV-with-detection for unrestricted agents, with the time given by Algorithm Bounded-energy-RV-with-detection for bounded-energy agents. This comparison is meaningful on the class of graphs for which both types of agents can achieve rendezvous with detection, i.e., for graphs of bounded size. Consider the class $C_{n}$ of graphs of size at most $n$, for some constant $n$, and consider $c$-bounded agents for some integer $c$ large enough to achieve rendezvous with detection on the class $C_{n}$ using Algorithm Bounded-energy-RV-with-detection. By Theorem 3.1, unrestricted agents can accomplish rendezvous with detection in time $O(P(n, \log \ell))$, i.e., since $n$ is constant, in time polylogarithmic in the smaller label. By contrast, by Theorem 4.1, bounded-energy agents can accomplish rendezvous with detection in time $O\left(\ell^{*}\right)$, i.e., linear in the larger label. It is natural to ask if this exponential gap in time, due to energy restriction, is unavoidable. The following lower bound shows that the answer to this question is yes. In fact, this lower bound holds even for the two-node graph, even with simultaneous start of the agents, and even for rendezvous without detection.

Theorem 4.2 Let $c$ be a positive constant. In the local beeping model, the time of rendezvous on the two-node graph of c-bounded agents with labels from the set $\{1, \ldots, M\}$ is $\Omega(\sqrt[c]{M})$. 
Proof. Consider any rendezvous algorithm $\mathcal{A}$ of $c$-bounded agents on the two-node graph. Suppose that labels of the agents can be drawn from the set $\{1, \ldots, M\}$ and that agents start simultaneously. Let $T$ be the worst-case meeting time over all pairs of labels from the set $\{1, \ldots, M\}$. For any label $L \in\{1, \ldots, M\}$, let $\Phi_{L}:\{1, \ldots, T\} \longrightarrow\{0,1\}$ denote the binary sequence of length $T$ with the following meaning. If agent with label $L$ executes alone algorithm $\mathcal{A}$ in the two-node graph (we call it the solo execution), then it moves in round $i$, if $\Phi_{L}(i)=1$, and it stays idle in round $i$, if $\Phi_{L}(i)=0$. For any label $L$, the function $\Phi_{L}$ is well-defined because in the two-node graph the history of the agent in any round is exactly the binary sequence describing the previous moves (i.e., the agent cannot "learn" anything from the environment during the navigation in this graph, as opposed to more complicated graphs in which it could learn degrees of visited nodes or port numbers by which it enters them). Hence a solo execution of algorithm $\mathcal{A}$ by an agent in the two node graph depends only on the label of the agent. Notice that, if there are two agents in this

graph, executing algorithm $\mathcal{A}$, then the behavior of each of them before the meeting is the same as in the solo execution.

Since agents are $c$-bounded, the number of values 1 in each function $\Phi_{L}$ is at most $c$. The number of such functions is

$$
\left(\begin{array}{l}
T \\
0
\end{array}\right)+\left(\begin{array}{l}
T \\
1
\end{array}\right)+\cdots+\left(\begin{array}{l}
T \\
c
\end{array}\right) \leq 1+T+T^{2}+\cdots+T^{c} \leq 2 T^{c} .
$$

If $2 T^{c}<M$, there would exist two labels $L_{1}, L_{2} \in\{1, \ldots, M\}$, for which $\Phi_{L_{1}}=\Phi_{L_{2}}$. Agents with these labels could not meet by round $T$, as they would move exactly in the same rounds until round $T$, hence in every round they would be at different nodes. It follows that $2 T^{c} \geq M$, hence $T \in \Omega(\sqrt[c]{M})$.

Theorem 4.2 implies that in the local beeping model, any rendezvous algorithm for bounded-energy agents must have time at least $\Omega\left(\sqrt[c]{\ell^{*}}\right)$, where $\ell^{*}$ is the larger label and $c$ is some constant. Theorems 3.1, 4.1 and 4.2 imply the following corollary.

Corollary 4.1 Rendezvous with detection of bounded-energy agents is feasible in the class of boundedsize graphs in the local beeping model, but its time must be exponentially larger than the best time of rendezvous with detection of unrestricted agents in this class of graphs.

\subsection{Bounded-energy agents in the global beeping model}

Our final result shows that in the stronger of our two models, i.e., the global beeping model, the lower bound on time proved in Theorem 4.2 does not hold anymore. In fact, we show that in this model, bounded-energy agents can meet with detection in the class of bounded-size graphs in time logarithmic in the smaller label. We will also prove that this time is optimal even in the two-node graph.

The high-level idea of the algorithm is to first break symmetry between the agents in time logarithmic in the smaller label, without making any moves, using the possibility of hearing the beeps of the other agent, wherever it is in the graph. This is the purpose of Procedure Symmetry-breaking 
in which agents beep or listen according to bits of their transformed label (the transformation will be explained below). Moreover, at the end of this procedure, both agents declare the same round to be red which permits them to synchronize in the next part of the algorithm.

After breaking symmetry, one of the agents remains idle and the other agent finds it using a bounded number of moves. This is done during Procedure Modified-beeping-exploration, when the moving agent performs exploration of the graph while beeping in every second round. This procedure is started by the moving agent in round red, simultaneously declared by both agents in Procedure Symmetry-breaking. Correct declaration of rendezvous is possible due to the distinction between hearing loud and soft beeps, and due to synchronization of agents which permits each of them to hear the beeps of the other agent.

We now proceed with the detailed description of the algorithm. We first define the following transformations of the label $L$ of an agent. Let $\left(c_{1} \ldots c_{k}\right)$ be the binary representation of the label $L$. Let $T_{1}(L)$ be the binary sequence $\left(01 c_{1} c_{1} c_{2} c_{2} \ldots c_{k} c_{k} 01\right)$, and let $T_{2}(L)$ be the result of replacing each bit 0 of $T_{1}(L)$ by the string (00) and each bit 1 by the string (10). Note that the length of the binary string $T_{2}(L)$ is $2(2 k+4) \in O(\log L)$.

The following procedure, executed by an agent with label $L$ and called upon the activation of the agent, does not involve any moves and permits to break symmetry between any two agents with different labels.

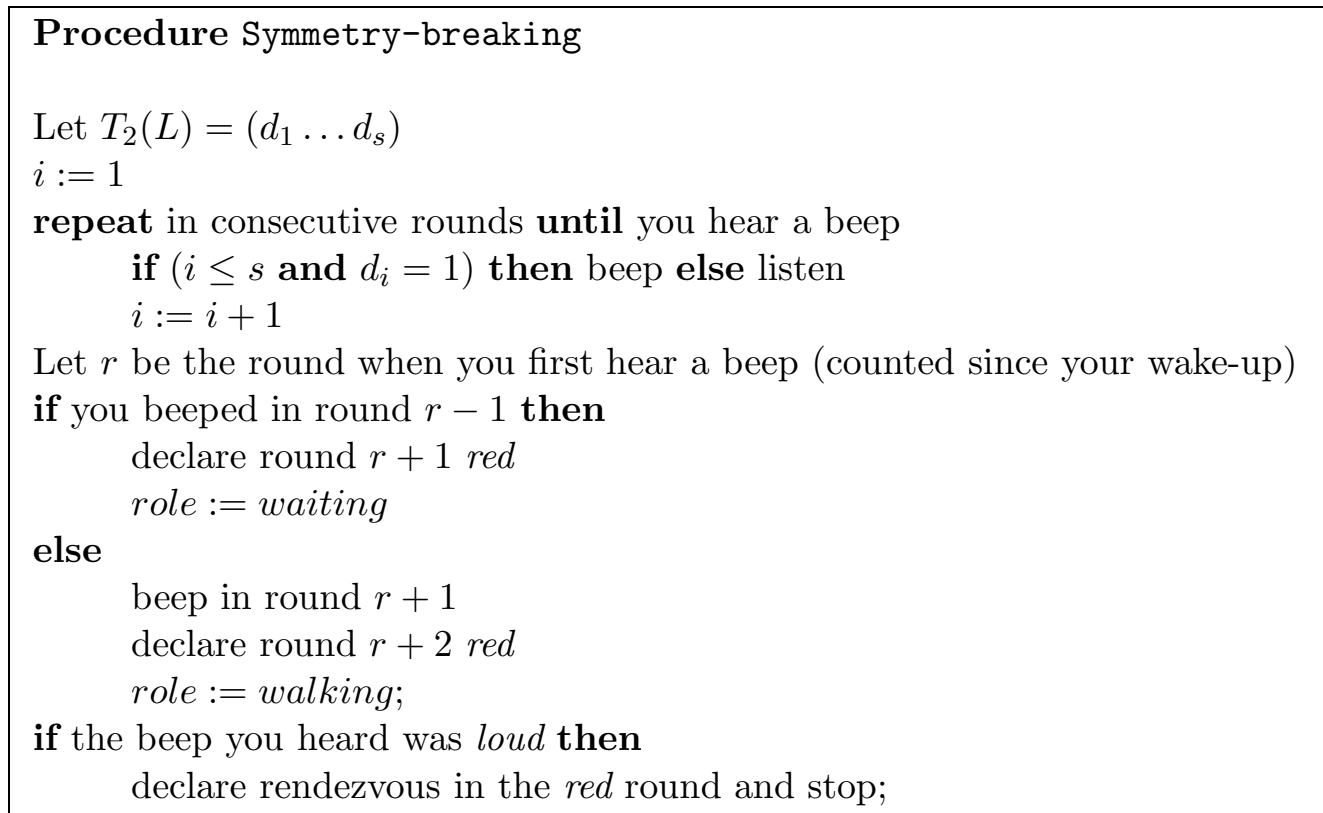

Lemma 4.1 Upon completion of Procedure Symmetry-breaking, both agents declare the same round to be red. For one of the agents round red is the next round after it heard a beep for the first time, and this agent sets role $:=$ waiting. For the other agent round red is two rounds after it heard a beep for the first time, and this agent sets role $:=$ walking. The round declared red is $O(\log \ell)$ rounds after the activation of the later agent, where $\ell$ is the smaller label.

Proof. We first prove that there exists a round $\xi$ in which both agents are present in the graph 
and one of the agents hears a beep. Let $L_{1}$ be the label of agent $A_{1}$ and let $L_{2}$ be the label of agent $A_{2}$. Consider three cases. In all of them we show a round $\xi$ as above, assuming that none of the agents heard a beep at any earlier round.

Case 1. Both agents are activated in the same round and have labels with binary representations of equal length.

Let $i$ be the first index for which strings $T_{2}\left(L_{1}\right)$ and $T_{2}\left(L_{2}\right)$ differ. Since agents are activated in the same round, the same round corresponds to index $i$ for both of them. In this round one of the agents beeps and the other agent listens, hence it hears a beep.

Case 2. Both agents are activated in the same round and have labels with binary representations of different length.

Without loss of generality, let $L_{1}$ be the label of smaller length. Let $T_{2}\left(L_{1}\right)=\left(d_{1} \ldots d_{s}\right)$. Let $\alpha$ be the common activation round of the agents and let $\gamma=\alpha+s-1$. Agent $A_{1}$ beeps in round $\gamma-1$ and listens in rounds $\gamma-3, \gamma-2$ and $\gamma$. Agent $A_{2}$ either listens in all rounds $\gamma-3, \gamma-2, \gamma-1$ and $\gamma$, or beeps in rounds $\gamma-3$ and $\gamma-1$ and listens in rounds $\gamma-2$ and $\gamma$. In both cases one of the agents hears a beep either in round $\gamma-3$ or $\gamma-1$.

Case 3. Agents are activated in different rounds.

Without loss of generality let $A_{1}$ be activated earlier and let $\alpha$ be its activation round. Let $\beta>\alpha$ be the activation round of $A_{2}$. Let $s$ be the length of $T_{2}\left(L_{1}\right)$.

If $\beta=\alpha+1$ or $\beta=\alpha+2$ then $A_{2}$ hears a beep in round $\alpha+2$. If $\beta=\alpha+3$ then $A_{2}$ hears a beep in round $\alpha+4$ because the first bit of the binary representation of every label $L$ is 1 , hence the 5 th bit of $T_{2}\left(L_{1}\right)$ is 1 .

Next suppose that $\alpha+4 \leq \beta \leq \alpha+s-8$. Consider three possibilities. If $\beta-\alpha$ is odd then agent $A_{2}$ beeps in round $\beta+2$ and agent $A_{1}$ listens in this round, hence it hears a beep. If $\beta-\alpha$ is divisible by 4 then agent $A_{2}$ listens in round $\beta$ and beeps in round $\beta+2$. Agent $A_{1}$ either listens in rounds $\beta$ and $\beta+2$ or beeps in these rounds. Hence one of the agents hears a beep in one of these rounds. The case of $\beta-\alpha=4 i+2$, for some integer $i$, is slightly more complicated. In this case divide all rounds, starting from round $\alpha$, into segments of size 2 . Round $\beta$ is in the beginning of such a segment. Segments correspond to bits of sequences $T_{1}\left(L_{1}\right)$ and $T_{1}\left(L_{2}\right)$. Let $T_{1}\left(L_{1}\right)$ be the binary sequence $\left(01 c_{1} c_{1} c_{2} c_{2} \ldots c_{k} c_{k} 01\right)$ and consider the final bits 01 of $T_{1}\left(L_{2}\right)$. Let $I$ be the segment corresponding to this bit 0 and let $J$ be the segment corresponding to this bit 1 . There are four possible situations:

1. The segment $I$ corresponds to some $c_{j}$ in $T_{1}\left(L_{1}\right)$ and the segment $J$ corresponds to $c_{j+1}$.

In this case the second copy of $c_{j+1}$ corresponds to a segment in which agent $A_{2}$ has already terminated and listens. If $c_{j+1}=0$ then $J$ corresponds to 0 in $T_{1}\left(L_{1}\right)$ and to 1 in $T_{1}\left(L_{2}\right)$. Hence $A_{1}$ hears a beep in the first round of this segment. If $c_{j+1}=1$ then in the first round of the segment following $J$ agent $A_{1}$ beeps and agent $A_{2}$ listens, hence it hears a beep.

2. The segment $I$ corresponds to $c_{k}$ in $T_{1}\left(L_{1}\right)$.

In this case $I$ corresponds to the second copy of $c_{k}$ in $T_{1}\left(L_{1}\right)$, and hence $J$ corresponds to 0 in $T_{1}\left(L_{1}\right)$ and to 1 in $T_{1}\left(L_{2}\right)$. Consequently, agent $A_{1}$ hear a beep in the first round of this segment. 
3. The segment $I$ corresponds to the final 1 in $T_{1}\left(L_{1}\right)$.

In this case $A_{2}$ hears a beep in the first round of segment $I$.

4. In the segment $I$ agent $A_{1}$ has already terminated and listens.

In this case, in segment $J$ corresponding to 1 in $T_{1}\left(L_{2}\right)$ agent $A_{1}$ also listens after termination. Hence, it hears a beep in the first round of this segment.

It follows that, whenever $\alpha+4 \leq \beta \leq \alpha+s-8$, one of the agents hears a beep at the latest in the round following the last round corresponding to a bit from $T_{2}\left(L_{2}\right)$ (or equivalently, at the latest in the round following the last round corresponding to a bit from $\left.T_{1}\left(L_{2}\right)\right)$.

If $\beta=\alpha+s-7$ then agent $A_{2}$ listens in round $\beta+1$ and beeps in round $\beta+2$, while agent $A_{1}$ either listens in both these rounds or beeps in round $\beta+1$ and listens in round $\beta+2$. Hence one of the agents hears a beep in one of these rounds. If $\beta=\alpha+s-6$ then agent $A_{2}$ beeps in round $\beta+2$ and agent $A_{1}$ listens in this round, hence it hears a beep. If $\beta=\alpha+s-5$ then agent $A_{2}$ beeps in round $\beta+2$ and agent $A_{1}$ listens in this round, hence it hears a beep. Finally, if $\beta>\alpha+s-5$, then agent $A_{2}$ beeps in round $\beta+4$ (because the first bit of the binary representation of every label $L$ is 1 , hence the 5 th bit of $T_{2}\left(L_{2}\right)$ is 1 ) and agent $A_{1}$ listens in this round (as it already concluded processing bits of $T_{2}\left(L_{1}\right)$ ), hence it hears a beep. This concludes the argument in Case 3 .

Hence one of the agents must always hear a beep in some round $\xi$. Moreover, our case analysis shows that round $\xi$ is at most $O(\log \ell)$ rounds after the activation of the later agent, where $\ell$ is the smaller label.

Let $\rho$ be the first round in which one of the agents hears a beep. Call this agent $A_{3}$ and the other agent $A_{4}$. Notice that $A_{3}$ could not beep in round $\rho-1$. Otherwise, agent $A_{4}$ that was already active in round $\rho-1$ and listened in this round (agents never beep in two consecutive rounds) would hear a beep in round $\rho-1$, contradicting the definition of round $\rho$. Hence, according to the procedure, agent $A_{3}$ beeps in round $\rho+1$ and declares round $\rho+2$ red. Agent $A_{4}$ which listens in round $\rho+1$ hears a beep in this round for the first time. Since it beeped in round $(\rho+1)-1$, it declares round $(\rho+1)+1$ red. Hence both agents declare the same round $\rho+2$ red.

For agent $A_{4}$, round red is the next round after it heard a beep for the first time, and this agent sets role $:=$ waiting. For agent $A_{3}$ round red is two rounds after it heard a beep for the first time, and this agent sets role $:=$ walking.

Since round $\rho$ is at most $O(\log \ell)$ rounds after the activation of the later agent, the same is true for the round declared red, which concludes the proof of the lemma.

We will also use a modified version of Procedure Beeping-exploration, described at the beginning of this section, for an integer parameter $n$.

Procedure Modified-beeping-exploration $(n)$

Let $\operatorname{EXP}(n)$ be the procedure described in Section 2 that permits exploration of all graphs of size at most $n$. Replace each round $r$ of $\operatorname{EXP}(n)$ by two consecutive rounds as follows. If in round $r$ of $\operatorname{EXP}(n)$ the agent takes port $p$ to move to node $w$, then in the first of the two replacing rounds the agent takes port $p$ to move to $w$ and beeps, and in the second replacing round it stays at $w$ and listens. 
Hence, in each of the two rounds replacing a round $r$ of $\operatorname{EXP}(n)$, the agent is at the same node in Procedure Modified-beeping-exploration $(n)$ as it is in Procedure $\operatorname{EXP}(n)$ in round $r$.

Below we give the pseudo-code of the algorithm executed by an agent with label $L$ in a graph of size at most $n$.

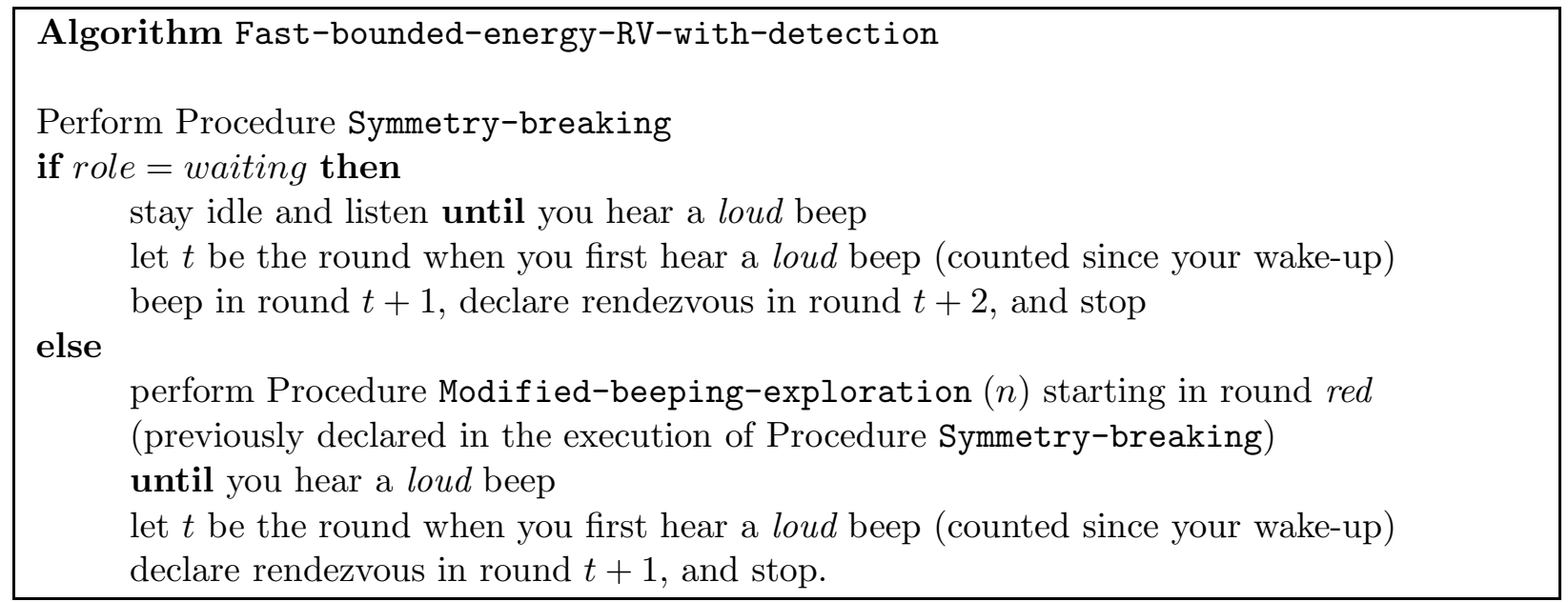

Theorem 4.3 For any positive integer constant $n$ there exists a positive integer $c$, such that Algorithm Fast-bounded-energy-RV-with-detection can be executed by c-bounded agents in any graph of size at most $n$, in the global beeping model. If two such agents with distinct labels execute this algorithm in such a graph, then they meet and simultaneously declare rendezvous in time $O(\log \ell)$ after the start of the later agent, where $\ell$ is the smaller label. This time is optimal, even in the two-node graph.

Proof. Let $n$ be a positive integer constant. In order to show the existence of the integer $c$, it is enough to show that the number of steps prescribed by the algorithm depends only on the constant $n$ (and not on the label $L$ of the agent). The agent moves only during the execution of Procedure Modified-beeping-exploration $(n)$, once every two rounds. Since a full execution of Procedure Modified-beeping-exploration $(n)$ takes $2 R(n)$ rounds (where $R(n)$ is the execution time of $\operatorname{EXP}(n))$ it is enough to take $c=R(n)$.

We now prove the correctness of the algorithm. By Lemma 4.1, both agents declare the same round to be red. In Procedure Symmetry-breaking, either both agents heard a loud beep, or they both heard a soft beep, because agents do not move during the execution of this procedure. If the beep they heard was loud then they are at the same node in round red and they correctly declare rendezvous in this round and stop. Hence we may assume that the beep they heard was soft. By Lemma 4.1, in round red one of the agents, call it $A_{1}$, has the variable role previously set to waiting, and the other agent, call it $A_{2}$, has the variable role previously set to walking. Agent $A_{1}$ stays idle forever at its starting node $v$. Let $\sigma$ be the first round in which agent $A_{2}$ arrives at node $v$. Such a round exists because $\operatorname{EXP}(n)$ explores the entire graph. Agent $A_{2}$ beeps in round $\sigma$. Agent $A_{1}$ hears a loud beep in this round for the first time. Hence it beeps in round $\sigma+1$ and declares rendezvous in round $\sigma+2$. Agent $A_{2}$ hears a loud beep for the first time in round $\sigma+1$. Hence it declares rendezvous in round $\sigma+2$. This concludes the proof of correctness. 
It remains to estimate the time between the start of the later starting agent and the declaration of rendezvous. By Lemma 4.1, the round declared red in Procedure Symmetry-breaking is $O(\log \ell)$ rounds after the activation of the later agent, where $\ell$ is the smaller label. Since $n$ is constant, $R(n)$ is also constant, and hence the duration of the execution of Procedure Modified-beeping-exploration $(n)$ is constant. It follows that the time between the activation of the later agent and the declaration of rendezvous is $O(\log \ell)$, where $\ell$ is the smaller label.

In order to prove that time $\Theta(\log \ell)$ is optimal, even in the global beeping model, consider the two-node graph $K_{2}$. For any integer $x>2$ and any algorithm $\mathcal{A}$ for rendezvous with detection in the global beeping model, working in time $t<(x-1) / 2$, we show two labels $L_{1}$ and $L_{2}$ with binary representations of length $x$, such that this algorithm fails if agents, placed at both nodes of $K_{2}$ and activated simultaneously, have these labels. In every round, the behavior of an agent can be described as a pair of bits, the first of which determines if the agent stays or moves in the given round, and the second of which determines if the agent beeps or does not beep in the given round. Hence, in an execution of an algorithm working in time $t$, there are $4^{t}$ possible behaviors of agents. In any execution in which an agent does not hear any beep, the behavior of the agent depends only on its label. There are $2^{x-1}$ labels with binary representations of length $x$ (each representation starts with 1$)$. Consequently, if $t<(x-1) / 2$, then $2 t<x-1$, and hence $4^{t}<2^{x-1}$, which implies that there are at least two labels with binary representations of length $x$, which induce identical behavior of the agents. None of such agents hears any beep, as they beep in exactly the same rounds, and they are at different nodes at all times. Hence the algorithm fails for them. This implies that rendezvous with detection must take time $\Omega(\log \ell)$, where $\ell$ is the smaller label.

\section{Conclusion}

We presented three algorithms of rendezvous with detection. The first two of them work even in the local beeping model: one for unrestricted agents in arbitrary graphs, and the other for bounded-energy agents in bounded-size graphs. We showed that in the latter case the meeting time of bounded-energy agents must be exponentially larger than the best time of rendezvous with detection of unrestricted agents. More precisely, in order to meet in bounded-size graphs, boundedenergy agents must use time polynomial in the larger label, while unrestricted agents can meet in time polylogarithmic in the smaller label. The third algorithm works for bounded-energy agents only in the global beeping model, but it is much faster: it enables such agents to perform rendezvous with detection in bounded-size graphs in time logarithmic in the smaller label, which is optimal.

Rendezvous with detection may be considered as a preprocessing procedure for other important tasks in graphs. One of them is the task of constructing a map of an unknown graph by an agent. It is well known that this task cannot be accomplished by a single agent operating in a graph, if it cannot mark nodes (e.g., a single agent cannot learn the size of an oriented ring). For the same reason it cannot be accomplished by two non-communicating agents, as they would not be aware of the presence of each other, and thus each of them would act as a single agent. By contrast, our algorithms of rendezvous with detection in the beeping model can serve, with a simple addition, to achieve map construction by the agents: the algorithm working for arbitrary agents can be used to 
accomplish this task in arbitrary graphs, and the algorithms working for bounded-energy agents can be used to accomplish this task in bounded-size graphs. This addition can be described as follows. Note that, in all our algorithms, at the time when agents declare rendezvous, symmetry between them is broken: in the case of algorithms in the local model, one of the agents heard two beeps at the meeting node, and the other agent heard only one beep, and in the case of the algorithm in the global model, one of the agents has role set to waiting and the other to walking. Hence agents can start simultaneously the following procedure in the round after rendezvous declaration. The first agent stays idle and acts as a stationary token, beeping in every second round, while the second agent silently executes exploration with a stationary token (at the end of which it acquires the map of the graph), cf. e.g., [6], replacing each exploration round by two rounds, in the first of which it moves as prescribed and in the second it stays idle. Beeps of the idle agent allow the circulating silent agent to recognize the token at each visit and complete exploration and map construction. At the end of the exploration, the second agent is with the first one and can inform it of the end of the exploration by beeping in the last round, in which the first agent is silent (listens). Then the roles of the agents may change to allow the previously idle agent to acquire the map in its turn. (Note that an agent cannot efficiently communicate the already acquired map due to the restrictive communication model.) 


\section{References}

[1] Y. Afek, N. Alon, Z. Bar-Joseph, A. Cornejo, B. Haeupler, F. Kuhn, Beeping a maximal independent set. Proc. 25th International Symposium on Distributed Computing (DISC 2011), LNCS 6950, 32-50.

[2] S. Alpern and S. Gal, The theory of search games and rendezvous. Int. Series in Operations research and Management Science, Kluwer Academic Publisher, 2002.

[3] E. Anderson and S. Fekete, Two-dimensional rendezvous search, Operations Research 49 (2001), 107-118.

[4] D. Baba, T. Izumi, F. Ooshita, H. Kakugawa, T. Masuzawa, Space-optimal rendezvous of mobile agents in asynchronous trees. Proc. 17th Int. Colloquium on Structural Information and Comm. Complexity, (SIROCCO 2010), LNCS 6058, 86-100.

[5] E. Bampas, J. Czyzowicz, L. Gasieniec, D. Ilcinkas, A. Labourel, Almost optimal asynchronous rendezvous in infinite multidimensional grids, Proc. 24th International Symposium on Distributed Computing (DISC 2010), LNCS 6343, 297-311.

[6] J. Chalopin, S. Das, A. Kosowski, Constructing a map of an anonymous graph: Applications of universal sequences, Proc. 14th International Conference on Principles of Distributed Systems (OPODIS 2010), 119-134.

[7] M. Cieliebak, P. Flocchini, G. Prencipe, N. Santoro, Distributed computing by mobile robots: Gathering, SIAM J. Comput. 41 (2012), 829-879.

[8] A. Cornejo, F. Kuhn, Deploying wireless networks with beeps, Proc. 24th International Symposium on Distributed Computing (DISC 2010), LNCS 6343, 148-162.

[9] J. Czyzowicz, A. Kosowski, A. Pelc, How to meet when you forget: Log-space rendezvous in arbitrary graphs, Distributed Computing 25 (2012), 165-178.

[10] A. Dessmark, P. Fraigniaud, D. Kowalski, A. Pelc. Deterministic rendezvous in graphs. Algorithmica 46 (2006), 69-96.

[11] Y. Dieudonné, A. Pelc, V. Villain, How to meet asynchronously at polynomial cost, SIAM J. Comput. 44 (2015), 844-867.

[12] P. Flocchini, G. Prencipe, N. Santoro, P. Widmayer, Gathering of asynchronous robots with limited visibility, Theoretical Computer Science 337 (2005), 147-168.

[13] P. Flocchini, N. Santoro, G. Viglietta, M. Yamashita, Rendezvous of two robots with constant memory, Proc. 20th Int. Colloquium on Structural Information and Comm. Complexity (SIROCCO 2013), LNCS 8179, 189-200.

[14] P. Fraigniaud, A. Pelc, Delays induce an exponential memory gap for rendezvous in trees, ACM Transactions on Algorithms 9 (2013), article 17. 
[15] S. Gilbert, C. Newport, The computational power of beeps, Proc. 29th International Symposium on Distributed Computing (DISC 2015), 31-46.

[16] M. Koucký, Universal traversal sequences with backtracking, Journal of Computer and System Sciences 65 (2002), 717-726.

[17] E. Kranakis, D. Krizanc, and P. Morin, Randomized Rendez-Vous with Limited Memory, Proc. 8th Latin American Theoretical Informatics (LATIN 2008), LNCS 4957, 605-616.

[18] E. Kranakis, D. Krizanc, N. Santoro and C. Sawchuk, Mobile agent rendezvous in a ring, Proc. 23rd Int. Conf. on Distr. Computing Systems (ICDCS 2003), 592-599.

[19] A. Miller and A. Pelc, Time versus cost tradeoffs for deterministic rendezvous in networks, Proc. 33rd Annual ACM Symposium on Principles of Distributed Computing (PODC 2014), 282-290.

[20] A. Pelc, Deterministic rendezvous in networks: A comprehensive survey, Networks 59 (2012), 331-347.

[21] O. Reingold, Undirected connectivity in log-space, Journal of the ACM 55 (2008).

[22] A. Ta-Shma and U. Zwick. Deterministic rendezvous, treasure hunts and strongly universal exploration sequences. Proc. 18th ACM-SIAM Symposium on Discrete Algorithms (SODA 2007), 599-608.

[23] J. Yu, L. Jia, D. Yu, G. Li, X. Cheng, Minimum connected dominating set construction in wireless networks under the beeping model, Proc. IEEE Conference on Computer Communications, (INFOCOM 2015), 972-980. 